\title{
Plurivascular Lesions in Patients with Peripheral Arterial Disease
}

\author{
Piroska Kelemen, Beáta Katalin Kolbert, Mónika Szabó, Hanga Kelemen, Achim Radu, \\ Alwina Ana Stan
}

University of Medicine and Pharmacy, Tîrgu Mureș, Romania

\section{CORRESPONDENCE}

Piroska Kelemen

Str. Gheorghe Marinescu nr. 38

540103 Tîrgu Mureș, Romania

Tel +40 265215551

E-mail: piroska_kelemen@yahoo.com

\section{ARTICLE HISTORY}

Received: 5 August, 2016

Accepted: 16 September, 2016
Beáta Katalin Kolbert • Str. Gheorghe Marinescu nr. 38, 540139 Tîrgu Mureș, Romania. Tel +40 265 215551

Mónika Szabó • Str. Gheorghe Marinescu nr. 38 540139 Tîrgu Mureș, Romania. Tel +40 265215551 Hanga Kelemen - Str. Gheorghe Marinescu nr. 38 , 540139 Tîrgu Mureș, Romania. Tel +40 265215551 Achim Radu • Str. Gheorghe Marinescu nr. 38, 540139 Tîrgu Mureș, Romania. Tel +40 265215551

Alwina Ana Stan • Str. Gheorghe Marinescu nr. 38 540139 Tîrgu Mures, Romania. Tel +40 265215551

\begin{abstract}
Introduction: Occlusive arterial disease, regardless of etiology, is a progressive chronic disease with multiple vessel involvement. The importance of obstructive arterial disease is that it leads to an increased mortality and morbidity of other cardiovascular disorders. Therefore, the presence of a lesion on a certain artery should lead to the identification of other lesions on the carotid and coronary arteries. The aim of the study was to assess the frequency and severity of lesions in peripheral arterial disease of different etiologies, and also to study its association with multivessel lesions at the level of the coronary tree and the carotid arteries. Material and methods: We performed a retrospective study on 177 patients with previously diagnosed peripheral artery disease that were admitted to the $2^{\text {nd }}$ Medical Clinic of the County Emergency Clinical Hospital of Tîrgu Mureș. The patients underwent evaluation of the peripheral artery disease (clinical, arterial Doppler), as well as of the coronary artery disease (clinical, ECG) and the carotid arteries (auscultation, Doppler Duplex ultrasound). The study population was divided into three groups: group 1 - diabetic arteriopathy $(n=79)$; group 2 - atherosclerotic obstructive arteriopathy ( $n=77$ ); group 3 - thromboangiitis obliterans ( $n=21)$. Results: The patients' age ranged between 61 and 70 years. Arterial occlusions were found in $87 \%$ of cases in group 1. Suboclussions were more frequent in diabetics (11.4\%). The artery, in which we found lesions in the highest percentage was the left femoral artery (group $1-68.3 \%$, group 2 $66.23 \%$, group $3-42.85 \%$ ). The second most often affected artery was the right femoral artery, with a $64.45 \%$ rate of involvement. In patients with thromboangiitis obliterans, the right anterior tibial artery was the most frequently affected (15.81\%). The most frequent coronary lesion was a stenosis of $30-69 \%$, in $35.02 \%$ of cases. Three-vessel coronary disease had an incidence of $44.2 \%$ in the atherosclerotic group, $34.2 \%$ in diabetics, and $23.8 \%$ in the thromboangiitis group. From the total rate of previously diagnosed myocardial infarction (MI) $-29.37 \%$ ( $n=$ 52), $50 \%$ were atherosclerotic, $44.23 \%$ diabetic, and $5.76 \%$ with thromboangiitis. At the level of the carotid system, $68.9 \%$ of patients were found to have stenoses under $70 \%$. From these, $48.4 \%$ were patients with diabetic arteriopathy, $44.3 \%$ with atherosclerotic disease. In $73.4 \%$ of cases, we found lesions at all three levels - in case of diabetic and nondiabetic arteriopathy and thromboangiitis, only $14.3 \%$ of patients had associated lesions. Conclusions: Atherosclerosis (diabetic and nondiabetic) and thromboangiitis obliterans are diseases with plurivascular involvement. It is important to diagnose concomitant coronary and carotid lesions in patients with chronic occlusive arterial illness, because they are often associated.
\end{abstract}

Keywords: peripheral artery disease, multivascular lesions, carotid lesions, coronary artery disease 


\section{INTRODUCTION}

Occlusive arterial disease, regardless of etiology, is a progressive chronic disease that may involve numerous arteries with plurisegmentary localization. ${ }^{1}$ Therefore, lesions that appear are not exclusive to the lower extremities. ${ }^{2}$ The importance of occlusive arterial disease is that it adds to the high mortality and morbidity of other cardiovascular disorders. ${ }^{3}$ For this reason, the presence of a lesion on a certain artery compels us to identify the presence of other lesions on the carotid and coronary arteries. ${ }^{4}$ The study of severity and/or frequency of arterial lesions is relevant in determining the prognosis of the disease. ${ }^{5}$

Taking into consideration the data obtained from the medical literature, our purpose was assess the frequency and severity of arterial lesions in peripheral arterial disease of different etiology.

At the same time, we studied plurivessel involvement at the level of the coronary and carotid arteries, both in patients with diabetic and nondiabetic atherosclerotic obstructive arteriopathy, and in those with thromboangiitis obliterans.

\section{MATERIAL AND METHODS}

In our retrospective study, we included 177 patients previously diagnosed with chronic occlusive arterial disease of the lower limbs. Patients have been admitted to the $2^{\text {nd }}$ Medical Clinic of the County Emergency Clinical Hospiral of Tîrgu Mureș in the period between November 2014 and March 2016.

The inclusion criteria were as follows:

Diagnosed chronic occlusive arterial disease (with intermittent claudication, cold lower limbs, paresthesia, pallor, tissue disorders, reduced or absent peripheral pulses, peripheral arterial murmur or angiographic signs of ischemia). Thus, all 177 patients included in our research were diagnosed with peripheral arterial disease. The purpose of our work was also to study the multiple vascular lesions in the coronary and carotid arteries. Lesions at this location were identified according to the algorithm described below, but we did not exclude patients with arteries in normal limits. The diagnosis of coronary artery disease was based on clinical (angina pectoris, dyspnea, palpitations, history of acute myocardial infarction) and electrocadiographical (ST-T segment changes) examination, as well as coronary angiography assessment. The carotid atherosclerotic lesions were confirmed by the presence of a history of transient ischemic attacks, arterial murmurs upon carotid auscultation, and also Doppler Duplex ultrasound of the carotid system.
The study population was grouped according to etiology in three groups:

1. Patients with diabetic arteriopathy $-\mathrm{n}=79$ (DA);

2. Patients with atherosclerotic obstructive arteriopathy $-\mathrm{n}=77$ (ATS);

3. Patients with thromboangiitis obliterans $-\mathrm{n}=$ 21(TAO).

\section{Study of peripheral arterial lesions}

To determine the severity of peripheral artery disease, using the ankle/brachial index measured with the continuous wave Doppler method, we divided the study population into three categories, taking into account the most severe lesions of the artery - occlusions, subocclusions (70-99\% stenosis) and stenosis of 30-69\%. We studied the severity of arterial lesions by counting occlusions and stenosis.

For the evaluation of coronary lesions, we used a similar method with the one for the lower limb lesion assessment. The presence of previous myocardial infarction and ischemic heart disease, revealed by the patients' medical history, resting ECG changes and coronary angiographic aspect indicated the presence of coronary atherosclerotic lesions. The most severe lesion of the artery was taken into account. We calculated the rate of normal coronary arteries, univascular, bi-vascular and three-vessel coronary artery disease.

We also assessed which coronary artery was more often involved, which was the most frequent infarction-related artery, and in every etiology group, which coronary arteries were more frequently found to have atherosclerotic lesions.

In order to assess the lesions of the carotids for each category, we used the same methods as in the evaluation of the coronary arteries. For each patient, the evaluation of the carotid arteries was performed by using color Duplex Doppler ultrasound, and the most severe lesions were noted.

We studied the three carotid arteries bilaterally: the common carotid artery (CCA), the internal carotid artery (ICA) and the external carotid artery (ECA). We also analyzed the etiology of each stenotic lesion.

To study multivessel atherosclerotic involvement, for each patient we evaluated the existence of concomitant lesions on the coronary and carotid arteries, according to the etiology. With this method, we formed two groups: group 1 - with multivascular involvement (concomitant peripheral, coronary and carotid lesions); group 2 - with peripheral and concomitant coronary/peripheral and concomitant carotid lesions. The data obtained were in- 


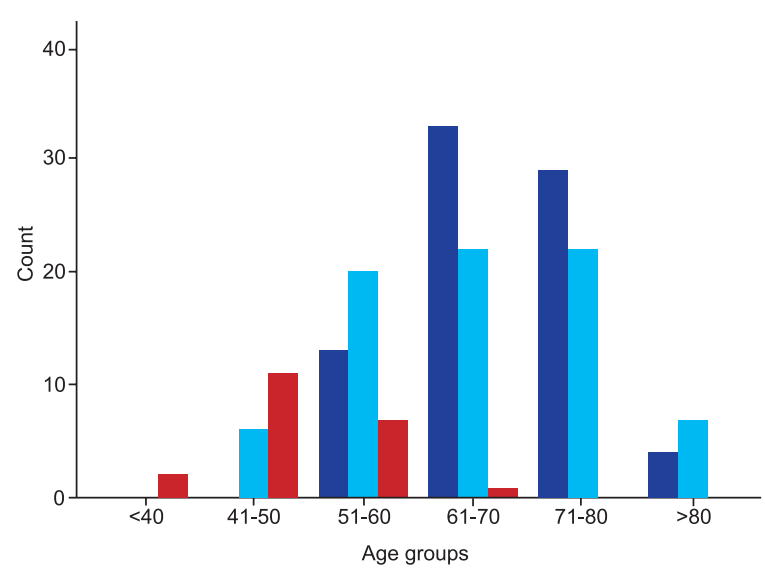

FIGURE 1. Age group distribution

troduced in Microsoft Excel 2007. Statistic processing and graphical representation was made with the following programs: GraphPad, MedCalc, IMB SPSS, using chi square and Anova tests.

\section{RESULTS}

\section{Lesions of limb arteries}

The age of the study population ranged between 61 and 70 years. In the thromboangiitis obliterans group $54.2 \%$ of the patients were between 41-50 years, and none of the patients was older than 70 years. In the atherosclerotic disease group, $57.2 \%$ of patients were between $61-80$ years. In the diabetic arteriopathy group, $41.8 \%$ of the patients were aged between $61-70$ years (Figure 1).

Comparing the etiology of arterial disease with age, we obtained a significant statistical correlation $(\mathrm{p}=0.0001)$.

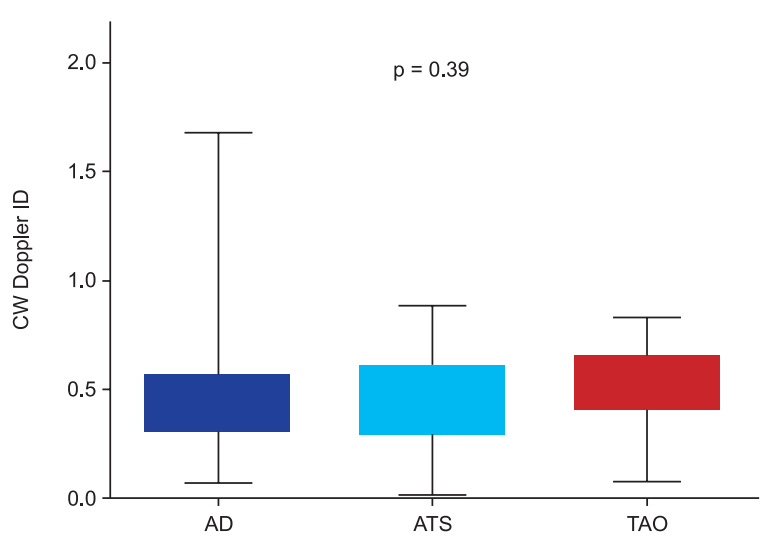

FIGURE 3. Peripheral arterial disease according to the Doppler index

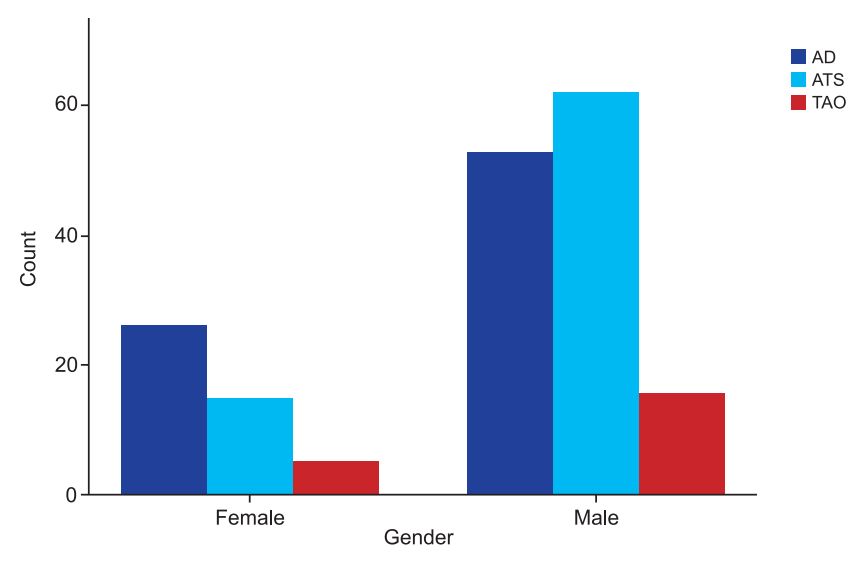

FIGURE 2. Patient distribution by gender

From the 177 patients included in our study, 25\% were females, of whom $56.5 \%$ were patients with diabetic arteriopathy. The majority of the patients were male $(n=131)$, from these $47.3 \%$ belonged to the atherosclerotic disease group (Figure 2).

Medium Doppler index, represented in Figure 3, is approximately equal (0.5) in all arterial diseases, with no regards to the involved artery. In the diabetic arteriopathy group, we found cases with values above 1 , which showed the presence of Monckeberg sclerosis. In the atherosclerotic group there were cases with pressure smaller than 10 $\mathrm{mmHg}$ (critical ischemia), which appear as values equal to 0 (Figure 3).

Taking into consideration the most severe lesion of the lower limb, occlusions were found in approximately equal proportions, in more than $80 \%$ of cases in all three groups, in atherosclerosis $-87 \%$.

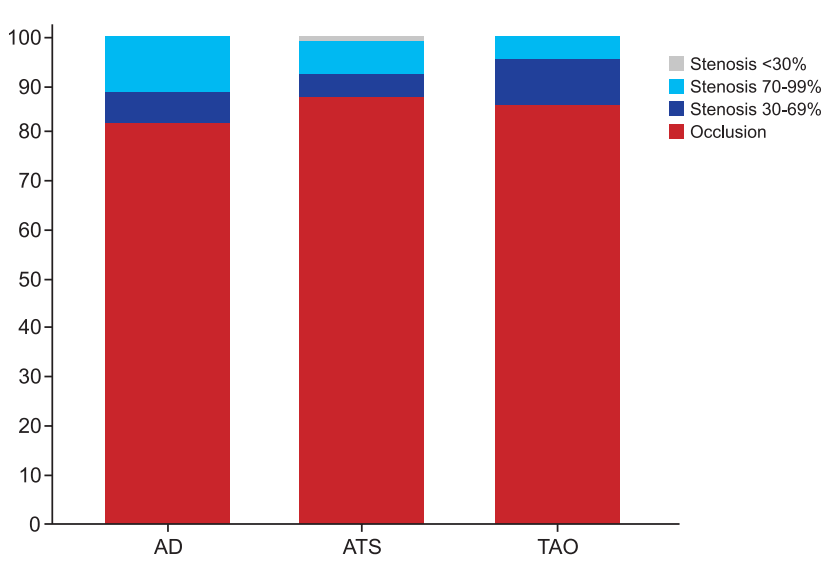

FIGURE 4. Peripheral artery disease according to the most severe lesion 
TABLE 1. Peripheral artery lesions by etiology

\begin{tabular}{lccc}
\hline & DA & ATS & TAO \\
\hline CIA R & $10.12 \%$ & $10.38 \%$ & $4.76 \%$ \\
EIA R & $11.39 \%$ & $15.58 \%$ & $0 \%$ \\
IIA R & $2.53 \%$ & $2.59 \%$ & $4.76 \%$ \\
CFA R & $5.06 \%$ & $9.09 \%$ & $0 \%$ \\
FA R & $67.8 \%$ & $64.93 \%$ & $42.85 \%$ \\
PFA R & $3.79 \%$ & $10.38 \%$ & $0 \%$ \\
PA R & $21.51 \%$ & $9.09 \%$ & $19.04 \%$ \\
ATA R & $16.45 \%$ & $6.49 \%$ & $47.6 \%$ \\
PTA R & $10.12 \%$ & $6.49 \%$ & $19.04 \%$ \\
CIA L & $5.06 \%$ & $16.88 \%$ & $4.76 \%$ \\
EIA L & $8.86 \%$ & $20.77 \%$ & $14.28 \%$ \\
IIA L & $0 \%$ & $2.59 \%$ & $4.76 \%$ \\
CFA L & $8.86 \%$ & $10.38 \%$ & $14.2 \%$ \\
FA L & $68.3 \%$ & $66.23 \%$ & $42.85 \%$ \\
PFA L & $2.53 \%$ & $7.79 \%$ & $4.76 \%$ \\
PA L & $25.31 \%$ & $10.38 \%$ & $19.04 \%$ \\
ATA L & $10.12 \%$ & $9.09 \%$ & $19.04 \%$ \\
PTA L & $11.39 \%$ & $7.79 \%$ & $4.76 \%$ \\
\hline
\end{tabular}

CIA - common iliac artery, EIA - external iliac artery, IIA - internal iliac artery, CFA - common femoral artery, FA - femoral artery, PFA - posterior femoral artery, PA - popliteal artery, ATA anterior tibial artery, PTA - posterior tibial artery, R - right, L - left

Subocclusions were more frequent in case of diabetes (11.4\%). Stenosis of less than $70 \%$ appeared more often in patients with thromboangiitis (Figure 4).

\section{The frequency of lesions in lower limb arteries}

In the case of patients with diabetes (DA) and those with atherosclerosis (ATS), the most frequently affected arteries were the right and left femoral arteries, in contrast with thromboangiitis obliterans, in which the most frequently affected artery was the right anterior tibial artery, followed by the two femoral arteries (Table 1).

In the entire group, the most affected artery was the left femoral artery, regardless of etiology, with lesions present in $65.53 \%$ of patients. Moreover, $68.53 \%$ of the lesions in the left femoral artery were occlusions.

Studying the left femoral artery among the three etiology groups, we observed a higher rate of occlusion in the atherosclerotic group, compared to the thromboangiitis group, in which the lesions were rarely present at the level of the femoral artery.

The second most often affected artery was the right femoral artery, with a $64.45 \%$ rate of involvement. At this level, $64.45 \%$ of the arteries were affected and $64.91 \%$ of patients showed lesions.
Occlusions were more frequent among the atherosclerotic disease group, and almost half of these patients presented occlusions. Both subocclusive stenosis and stenosis of lower grades (30-69\% and less than $30 \%$ ) were found to be more frequent in patients with diabetic arteriopathy. Patients with thromboangiitis obliterans were less affected, but there was a higher percentage of occlusions and we observed more arteries that were normal. The results did not show any statistically significant differences $(p=0.109)$.

The right anterior tibial artery was the most frequently affected artery in patients with thromboangiitis obliterans. It was affected in $15.81 \%$ of patients, from which $71.42 \%$ presented obstructions. In the thromboangiitis group, $35.71 \%$ presented both obstruction and stenosis in higher percentages. Among diabetics, we observed a low frequency of involvement of the right tibial artery compared to the thromboangiitis group. In the atherosclerosis group, $100 \%$ presented obstructions (Table 2). The difference between the three etiology groups regarding the involvement of the right tibial artery was statistically significant $(\mathrm{p}=0.002)$.

\section{Lesions of coronary arteries}

The most frequent coronary lesion was a stenosis of 30 $69 \%$, found in $35.02 \%$ of cases. From the total number of patients presenting with coronary artery lesions, $48.4 \%$ had atherosclerotic disease and $45.2 \%$ had diabetic arteriopathy. In the diabetic group, $48.4 \%$ of patients presented coronary occlusions, and $6.3 \%$ subocclusions. In the thromboangiitis group, $47.6 \%$ of the total coronary arteries that were evaluated did not have significant lesions (Figure 5). The difference between the groups was not statistically significant $(\mathrm{p}=0.10)$.

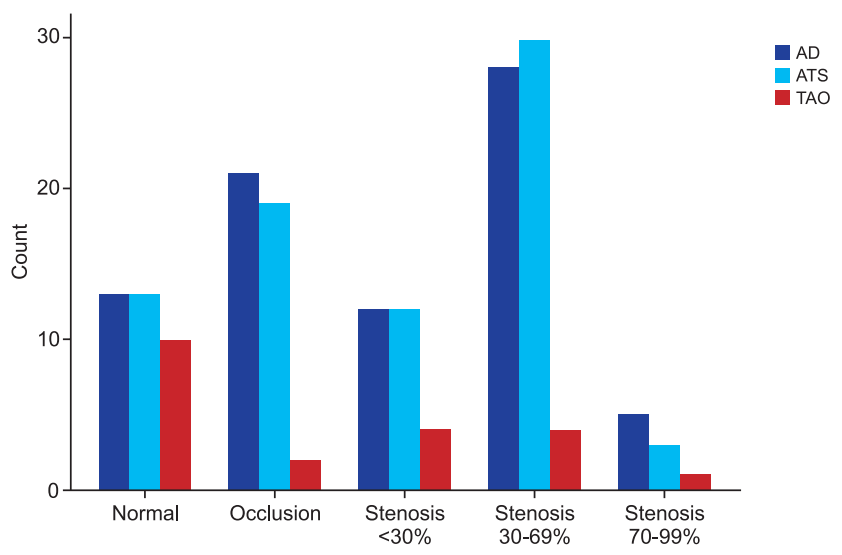

FIGURE 5. The severity of coronary arteries lesions in the studied groups 
TABLE 2. Right anterior tibial artery lesions by etiology

\begin{tabular}{|c|c|c|c|c|c|}
\hline & & \multicolumn{3}{|c|}{ Diagnosis } & \multirow[t]{2}{*}{ Total } \\
\hline & & DA & ATS & TAO & \\
\hline \multirow[t]{3}{*}{ Normal } & Count & 66 & 72 & 11 & 149 \\
\hline & $\%$ within ATA right & $44.3 \%$ & $48.3 \%$ & $7.4 \%$ & $100 \%$ \\
\hline & $\%$ within Diagnostic & $83.5 \%$ & $93.5 \%$ & $52.4 \%$ & $84.2 \%$ \\
\hline \multirow[t]{3}{*}{ Occlusion } & Count & 7 & 5 & 8 & 20 \\
\hline & $\%$ within ATA right & $35 \%$ & $25 \%$ & $40 \%$ & $100 \%$ \\
\hline & $\%$ within Diagnostic & $8.9 \%$ & $6.5 \%$ & $38,1 \%$ & $11.3 \%$ \\
\hline \multirow[t]{3}{*}{ Stenosis <30\% } & Count & 3 & 0 & 0 & 3 \\
\hline & $\%$ within ATA right & $100 \%$ & $0 \%$ & $0 \%$ & $100 \%$ \\
\hline & $\%$ within Diagnostic & $3.8 \%$ & $0 \%$ & $0,0 \%$ & $1,7 \%$ \\
\hline \multirow[t]{3}{*}{ Stenosis 30-69\% } & Count & 1 & 0 & 1 & 2 \\
\hline & $\%$ within ATA right & $50 \%$ & $0 \%$ & $50 \%$ & $100 \%$ \\
\hline & $\%$ within Diagnostic & $1.3 \%$ & $0 \%$ & $4.8 \%$ & $1.1 \%$ \\
\hline \multirow[t]{3}{*}{ Stenosis 70-99\% } & Count & 2 & 0 & 1 & 3 \\
\hline & $\%$ within ATA right & $66.7 \%$ & $0 \%$ & $33.3 \%$ & $100 \%$ \\
\hline & $\%$ within Diagnostic & $2.5 \%$ & $0 \%$ & $4.8 \%$ & $1.7 \%$ \\
\hline \multirow[t]{3}{*}{ Total } & Count & 79 & 77 & 21 & 177 \\
\hline & $\%$ within ATA right & $44.6 \%$ & $43.5 \%$ & $11.9 \%$ & $100 \%$ \\
\hline & $\%$ within Diagnostic & $100 \%$ & $100 \%$ & $100 \%$ & $100 \%$ \\
\hline
\end{tabular}

Three-vessel coronary disease had an incidence of $44.2 \%$ in the atherosclerotic group, $34.2 \%$ in the diabetic group, and $23.8 \%$ in the thromboangiitis group.

Taking into consideration lesions of each coronary artery separately, we observed that the most affected was the circumflex artery in $60.45 \%$ of cases, the majority (63.3\%) being of atherosclerotic etiology.

Looking at the frequency of the lesions, the second was the anterior descendent artery, in 105 cases $(59.32 \%)$. This artery was also more frequently affected in the atherosclerosis group. The right coronary artery was the least affected (54.54\%), with most cases within the atherosclerosis group.

The most frequently involved artery in both the diabetic and the thromboangiitis group was the circumflex artery, and in the atherosclerotic group, the anterior descendant artery was the most affected one (Table 3).

Regarding the rate of myocardial infarction (MI), our results showed that from the total number of patients pre-

TABLE 3. Coronary lesions by etiology

\begin{tabular}{lccc}
\hline & DA & ATS & TAO \\
\hline RCA & $44.3 \%$ & $54.54 \%$ & $33.33 \%$ \\
LAD & $59.49 \%$ & $67.53 \%$ & $33.33 \%$ \\
CXA & $62.02 \%$ & $63.63 \%$ & $42.85 \%$ \\
\hline
\end{tabular}

RCA - right coronary artery, LAD - left anterior descendant, CXA - Circumflex artery, DA diabetic arteriopathy, ATS - atherosclerosis, TAO - thromboangiitis obliterans senting MI $-29.37 \%(n=52), 50 \%$ were included in the atherosclerotic group, $44.23 \%$ in the diabetic arteriopathy, and $5.76 \%$ in the thromboangiitis group. Inversely, from the total number of diabetic patients, $29.11 \%$ presented a history of MI, $33.76 \%$ from the atherosclerotic group and only $14.28 \%$ of patients with thromboangiitis had a previously diagnosed MI.

\section{Carotid artery lesions}

At the level of the carotid system, $68.9 \%$ of patients were found to have stenoses under $70 \%$. From these, $48.4 \%$

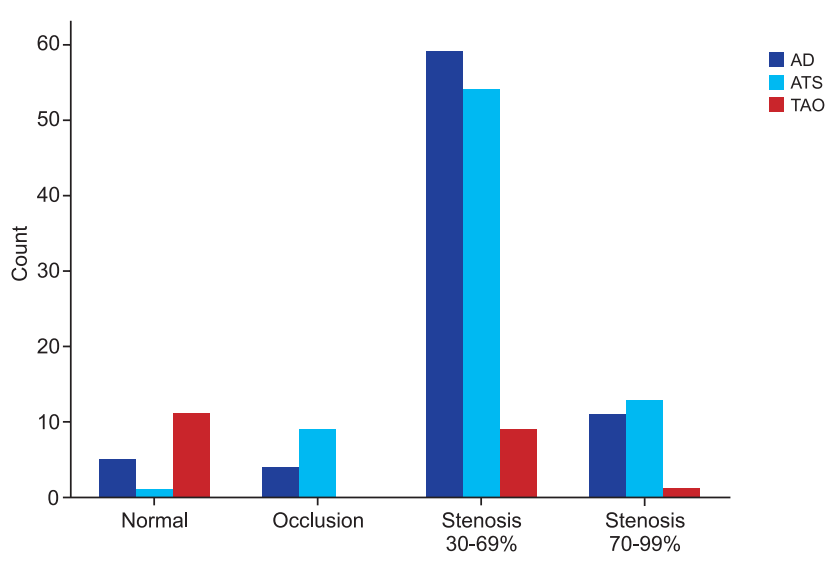

FIGURE 6. The severity of carotid lesions 
TABLE 4. Carotid artery lesions by etiology

\begin{tabular}{lccc}
\hline & DA & ATS & TAO \\
\hline CCA R & $32.91 \%$ & $42.85 \%$ & $23.8 \%$ \\
ICA R & $41.77 \%$ & $38.96 \%$ & $4.76 \%$ \\
ECA R & $13.92 \%$ & $3.89 \%$ & $0 \%$ \\
CCA L & $41.77 \%$ & $45.45 \%$ & $38.09 \%$ \\
ICA L & $41.77 \%$ & $44.15 \%$ & $0 \%$ \\
ECA L & $13.92 \%$ & $5.19 \%$ & $0 \%$ \\
\hline
\end{tabular}

CCA - common carotid artery, ICA - internal carotid artery, ECA - external carotid artery, R right, $L-$ left

were patients with diabetic arteriopathy and $44.3 \%$ with atherosclerotic disease. From the total number of patients, 7.3\% presented occlusions, most often within the atherosclerotic group. In the thromboangiitis group, we did not find any occlusion. Subocclusions appeared less frequently than occlusions, but with similar distribution. From subocclusion lesions, only one case belonged to the thromboangiitis group (Figure 6).

In our study, $61.6 \%$ of patients had two carotids being affected concomitantly. From these, $48.6 \%$ were with atherosclerosis, $46.8 \%$ were diabetics, and $4.6 \%$ were with thromboangiitis obliterans. Concomitant involvement of three or more carotids had a very low prevalence, affecting only $8.5 \%$ of patients, and they appeared more often in the diabetic arteriopathy group, the difference between the groups being statistically significant $(\mathrm{p}=0.0001)$.

Studying the carotids separately, regardless of etiology, we observed that most affected arteries were the left common carotid artery in $42.93 \%$, and the left internal carotid artery in $37.8 \%$ of patients. These two arteries were most often affected by atherosclerosis as well. In diabetic arteriopathy, the above-mentioned arteries, together with the right internal carotid artery were affected most frequently.

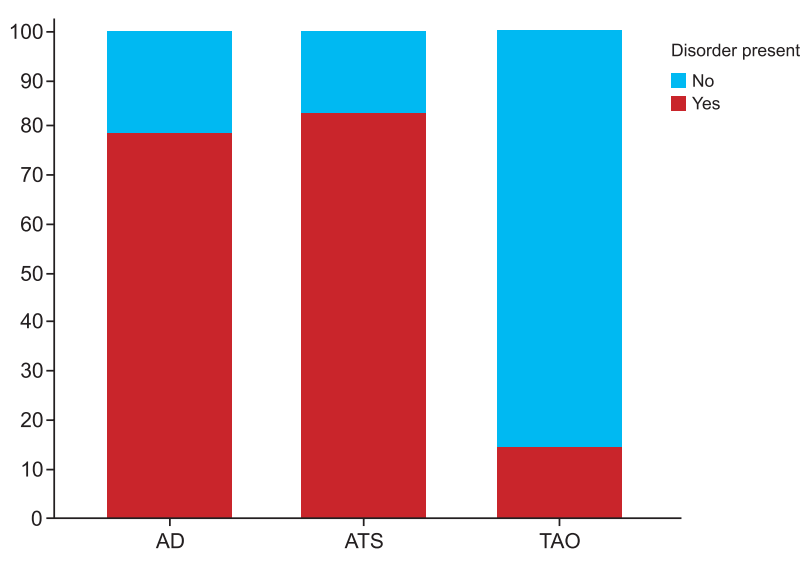

FIGURE 7. The presence of multivascular involvement
In the thromboangiitis group, the most often affected carotid was the left common carotid artery (Table 4).

\section{Multivascular disorder - association of lesions}

From the 130 patients with multivascular disorder, we found lesions at all three levels in $73.4 \%$ of cases. In the diabetic and nondiabetic arteriopathy, the frequency was approximately equal. In contrast, we found an association of lesions in only $14.3 \%$ of patients with thromboangiitis obliterans (Figure 7).

\section{DISCUSSIONS}

In diabetic arteriopathy, there were more arterial stenoses compared to other etiologies, affecting most often both the femoral and distal arteries.

In atherosclerotic disease,we found more frequent occlusions of the large arteries: iliac and femoral arteries. In the diabetic arteriopathy and thromboangiitis groups, the distal arteries were more frequently affected. Our results match with data from the medical literature. ${ }^{6-8}$

From our data, we can state that the most severely affected coronaries were found in the diabetes group. The same result was found in similar studies. ${ }^{9}, 10$ The group with thromboangiitis presented a low frequency of coronary lesions. In all patients with arterial disease, the most frequent were concomitant lesions on all three coronary arteries. In patients with atherosclerotic disease the most frequently and severely affected were the branches of left coronary artery. In patients with diabetic arteriopathy, subocclusive stenoses appeared more frequently.

Studying the three levels (peripheral, coronary, carotid), we found the lowest number of occlusions at the carotid level.

Occlusions have been most frequently described in atherosclerotic disease. ${ }^{11}$ Subocclusive lesions appeared in almost equal frequency in patients with diabetic and nondiabetic arteriopathy. In the thromboangiitis group we did not find any carotid involvement. Therefore, we can conclude that atherosclerosis affects more frequently one or two arteries from the carotid system, in diabetes it affects three or more. In accordance with literature data, diabetes causes multisegmentary serial lesions. ${ }^{10}$ In $57.1 \%$ of thromboangiitis cases no lesions were described on the carotid system.

In all studied patients, regardless of the etiology, we noticed that similarly with other studies, the left carotid system was more often affected than the right. ${ }^{10}$ In each etiology group, the lesions of the common carotid artery 
were important. Lesions of the internal carotid artery were the most important in atherosclerosis and diabetes. ${ }^{11}$

In our study, the highest frequency of multivessel involvement was observed in case of atherosclerosis, followed by diabetes, which concurs with results found in the literature. ${ }^{12-14}$ In thromboangiitis patients, multivascularity was more rare.

\section{CONCLUSIONS}

In the patients included in the study, regardless of etiology, the femoral arteries have been the most frequently affected. In diabetic arterial disease, and mostly in thromboangiitis obliterans, the distal arteries were more frequently affected.

Analyzing the coronary system in patients with chronic obstructive arterial disease, we observed more severe lesions in diabetics. Ischemic heart disease was more frequently found in the atherosclerotic disease group. In thromboangiitis, the coronary system was more rarely affected than in the other groups. Myocardial infarctions occurred more often with involvement of the left coronary artery branches.

The carotid system did not present very severe lesions. The left carotid system was the most frequently affected. In atherosclerotic disease, there were more occlusions. Concomitant lesions of more arteries from the carotid system were significantly more frequent in the diabetic group. In the thromboangiitis group the carotid lesions were more rare than the coronary lesions.

Concomitant disorders: peripheral arteries, coronaries and carotids were often present (73.4\%). In diabetic and nondiabetic arterial disease, the frequency of multivascular lesions was approximately equal, around $80 \%$, being much lower in patients with thromboangiitis.

Atherosclerosis (diabetic and nondiabetic) and thromboangiitis obliterans are diseases with plurivascular involvement. It is important to diagnose concomitant coro- nary and carotid lesions in patients with chronic occlusive arterial illness, because they are often associated.

\section{CONFLICT OF INTEREST}

Nothing to declare.

\section{REFERENCES}

1. Barbarash OL, Zykov MV, Pecherina TB, et al. The Prognostic Value of Peripheral Artery Diseases in Patients with ST-Segment Elevation Myocardial Infarction. Dis Markers. 2013;35(6):877-882.

2. Yakubov S. Polyvascular atherosclerotic disease: recognizing the risks and managing the syndrome. Curr Med Res Opin. 2009;25(11):2631-2641.

3. Alahdab F, Wang AT, Elraiyah TA, et al. A systematic review for the screening for peripheral arterial disease in asymptomatic patients. J Vasc Surg. 2015;61(3S):42S-53S

4. Folsom AR, Kronmal RA, Detrano RC, et al. Coronary Artery Calcification Compared with Carotid Intima-Media Thickness in Prediction of Cardiovascular Disease Incidence: The Multi-Ethnic Study of Atherosclerosis (MESA). Arch Intern Med. 2008;168(12):1333-1339.

5. Espinola-Klein C, Rupprecht HJ, Blankenberg S, et al. Manifestations of atherosclerosis in various vascular regions. Similarities and differences regarding epidemiology, etiology and prognosis. Med Klin (Munich). 2002;97(4):221-228.

6. Aboyans V, Ho E, Denenberg JO, et al. The association between elevated ankle systolic pressures and peripheral occlusive arterialdisease in diabetic and nondiabetic subjects. J Vasc Surg. 2008;48(5):1197-1203.

7. Lundberg $\mathrm{C}$, Hansen $\mathrm{T}$, Ahlström $\mathrm{H}$, et al. The relationship between carotid intima-media thickness and global atherosclerosis. Clin Physiol Funct Imaging. 2014;34(6):457-462

8. Lee MS, Rha SW, Han SK, et al. Comparison of diabetic and non-diabetic patients undergoing endovascular revascularization for peripheral arterial disease. J Invasive Cardio. 2015;27(3):167-171.

9. Bosevski M. Peripheral arterial disease and diabetes. Prilozi. 2012;33(1):6578.

10. Stam-Slob MC, van der Graaf Y, de Borst GJ, et al. The Effect of Type 2 Diabetes on Recurrent Major Cardiovascular Events for Patients With Symptomatic Vascular Disease at Different Locations. Diabetes Care. 2015;38(8):1528-1535

11. Bosevski M. Carotid artery disease in diabetic patients. Prilozi. 2014;35(3):149-162.

12. Imori Y, Akasaka T, Ochiai T, et al. Co-existence of carotid artery disease, renal artery stenosis, and lower extremity peripheralarterial disease in patients with coronary artery disease. Am J Cardiol. 2014;113(1):30-35.

13. Fernández-Friera L, Peñalvo JL, Fernández-Ortiz A, et al. Prevalence, Vascular Distribution, and Multiterritorial Extent of Subclinical Atherosclerosis in a Middle-Aged Cohort: The PESA (Progression of Early Subclinical Atherosclerosis) Study. Circulation. 2015;131(24):2104-2113.

14. Poredos P, Jug B. The prevalence of peripheral arterial disease in high risk subjects and coronary or cerebrovascular patients. Angiology. 2007;58(3):309-315. 